\title{
A Well-Designed Parameter Estimation Method for Lifetime Prediction of Deteriorating Systems with Both Smooth Degradation and Abrupt Damage
}

\author{
Chuanqiang Yu and Cheng Jiang \\ High-Tech Institute of Xian, Xian, Shaanxi 710025, China \\ Correspondence should be addressed to Chuanqiang Yu; chuanqiangyu@126.com
}

Received 26 November 2014; Accepted 5 January 2015

Academic Editor: Gang Li

Copyright @ 2015 C. Yu and C. Jiang. This is an open access article distributed under the Creative Commons Attribution License, which permits unrestricted use, distribution, and reproduction in any medium, provided the original work is properly cited.

Deteriorating systems, which are subject to both continuous smooth degradation and additional abrupt damage due to a shock process, can be often encountered in engineering. Modeling the degradation evolution and predicting the lifetime of this kind of systems are both interesting and challenging in practice. In this paper, we model the degradation trajectory of the deteriorating system by a random coefficient regression (RCR) model with positive jumps, where the RCR part is used to model the continuous smooth degradation of the system and the jump part is used to characterize the abrupt damage due to random shocks. Based on a specified threshold level, the probability density function (PDF) and cumulative distribution function (CDF) of the lifetime can be derived analytically. The unknown parameters associated with the derived lifetime distributions can be estimated via a well-designed parameter estimation procedure on the basis of the available degradation recordings of the deteriorating systems. An illustrative example is finally provided to demonstrate the implementation and superiority of the newly proposed lifetime prediction method. The experimental results reveal that our proposed lifetime prediction method with the dedicated parameter estimation strategy can get more accurate lifetime predictions than the rival model in literature.

\section{Introduction}

Over the past decade, prognostics and health management (PHM) has been well recognized as an effective and systematic discipline of enhancing reliability, determining the advent of failure, and reducing operating risk through managerial activities [1-5]. The main contents of PHM can be classified into the following two parts, that is, "prognostics" and "health management." Specifically, the former is dedicated to finding the mean or distributions (e.g., PDF and $\mathrm{CDF}$ ) of the system's lifetime, while the latter aims at taking appropriate decisions (such as spare part ordering and maintenance scheduling) on the basis of the predicted lifetime distributions [6-10]. As a result, lifetime prediction technique constitutes the central component of PHM program in practical implementation $[1,7,11]$. The existing lifetime prediction methods in literature can be broadly divided into physicsbased and data-driven methods [12]. Physics-based methods aim to predict the system's lifetime on the basis of the physics of the system's underlying failure mechanisms, and datadriven methods achieve lifetime predictions relying mainly on the condition monitoring data (e.g., wear, crack length, vibration, capacitance, drift rate of an electronic device, and light intensity of a light emitting diode), which can reflect the health condition of a practical system. Compared with the former, the latter has become the mainstream of the current lifetime prediction practices [1, 12]. This is mainly because (1) it is typically difficult, or even impossible, to capture the physics of failure of an engineering system in practice, especially for the complicated or large-scale systems operating under time-varying environments, and (2) the condition monitoring data which are highly correlated with the underlying health condition (equivalently, lifetime) of an engineering system can be measured and collected easier and easier, owing to the rapid development of sensor and instrumentation techniques. Thus, the data-driven lifetime prediction methods are more appealing and have gained much attention in the past years, [10-13]. 
In literature, it is well accepted that the degradation process of a practical system is typically stochastic and uncertain, because of the time-varying working loads and dynamic operating environments, and therefore, stochastic models are suggested to be utilized to characterize the degradation trajectory of a deteriorating system [7, 10-13]. This is mainly because not only the point prediction of the system's lifetime but also the uncertainty associated with the predicted lifetime distributions can be obtained based on a stochastic model for degradation modeling. RCR is one of the most frequently used stochastic degradation models in both industrial and academic domains [11]. The fundamental rationale of the RCR-based degradation models is to model the system's degradation evolution based on the regression model with a random coefficient, where the random coefficient is used to characterize the heterogeneity among deteriorating systems of the same kind [11, 14]. As far as the authors know, Lu and Meeker were the first to use the RCR-based degradation model in lifetime prediction area [14]. Up to now, the RCR models have been widely used in degradation modeling and lifetime prediction practices, such as fatigue crack data [14], bearings [15], vacuum fluorescent displays data [16], and gyroscopes [17].

However, most of the RCR-based degradation modeling and lifetime prediction methods in literature share a common assumption that the deteriorating systems should degrade smoothly. Taking both the continuous smooth degradation and the abrupt damage of random shocks into consideration, Peng et al. [18] proposed a degradation modeling method for lifetime prediction, where the continuous smooth degradation part was modeled by a RCR model. Based on the framework developed in [18], extensions and applications were widely made in [19-22] during the recent years. It should be noted that the parameters in the degradation model of [18] and its followers were all assumed to be known as prior information or can be appointed by subjective hypotheses, and no parameter estimation procedure was provided. As it is known to all, the choosing of the values of these unknown parameters plays a key role in the predicted lifetime distributions, and thus, if these parameters are misspecified, then the predicted lifetime distributions may be unreasonable. To surmount this problem, this paper proposed a dedicated parameter estimation method for lifetime prediction of deteriorating systems subjected to both continuous smooth degradation and additional abrupt damage due to a shock process. To be specific, we model the degradation trajectory of this kind of system by a RCR model with positive jumps, where the RCR model is used to track the system's smooth degradation and the jumps part is used to characterize the abrupt damage due to random shocks. Based on a specified threshold level, the analytical PDF and CDF of lifetime can be derived. To estimate the unknown parameters associated with the lifetime distributions, a well-designed parameter estimation method is formulated by using the maximum likelihood estimation (MLE) strategy. The effectiveness and superiority of the newly proposed lifetime prediction method are finally validated by an illustrative example.

The remainder of this paper is organized as follows. Section 2 describes the problem to be explored in this paper.
Section 3 is devoted to deriving the PDF and CDF of the lifetime of the deteriorating systems. In Section 4, the parameter estimation procedure is specified. An illustrative example is provided in Section 5 for demonstration. Section 6 concludes the paper with possible directions for future researches.

\section{Problem Description}

For a deteriorating system subjected to both continuous smooth degradation and additional abrupt damage due to a shock process, its total degradation evolution can be characterized by the following additive model:

$$
X(t)=X_{a}(t)+X_{S}(t),
$$

where $X(t)$ represents the system's degradation at time $t$, $X_{a}(t)$ is the continuous smooth degradation at time $t$, and $X_{S}(t)$ is the accumulated abrupt damage till time $t$ due to random shocks.

Remark 1. We can observe from (1) that the actual degradation $X(t)$ is the summation of the continuous smooth degradation $X_{a}(t)$ at time $t$ and the accumulated abrupt damage till time $t$. In other words, both continuous smooth degradation and accumulated abrupt damage result in the system's actual degradation.

Specifically, we choose the following specific model [18] to characterize the degradation of the deteriorating system considered in this paper:

$$
X(t)=\alpha+\beta t+\sum_{k=0}^{Z(t)} L_{k},
$$

where $X_{a}(t)=\alpha+\beta t$ and $X_{S}(t)=\sum_{k=0}^{Z(t)} L_{k}$ correspond to (1). $\alpha=X(0)$ is the initial degradation of a system, and without loss of generality, $\alpha$ is assumed to be zero in this paper [7, $12,13,17,18] . \beta \sim N\left(\mu_{\beta}, \sigma_{\beta}^{2}\right)$ represents the degradation rate of the deteriorating system, and the randomness of $\beta$ is used to characterize the heterogeneity over a class of systems [1518]. $Z(t)$ is a Poisson process representing the number of the random shocks up to time $t$ with rate $\tau$, and $L_{k}\left(k \in N^{+} \cup\{0\}\right)$ represents the abrupt damage due to the $k$ th shock with $L_{0}=$ 0 . We further assume that $L_{k}$ is a positive random variable with independent identically distributed (IID) PDF, $f_{L}(l ; \xi)$, and $\xi$ is the unknown parameters. As a consequence, $X_{S}(t)$ constitutes a compound Poisson process.

From the degradation model defined in (1) and (2), the lifetime $T$ of the deteriorating system can be defined as

$$
T=\inf \{t: X(t) \geq w \mid X(0)<w\},
$$

where $w$ represents the threshold level, which can be specified by the industrial standards, the expert knowledge, and so forth [11-18].

Based on the definitions in (1) (3), we can obtain the issues that need to be studied in this paper. First, deriving the PDF and CDF of the lifetime defined in (3). Second, estimating the unknown parameters associated with the obtained lifetime distributions. 


\section{Derivation of the Lifetime Distributions}

This section is devoted to deriving the lifetime distributions defined in (3). According to (1) (3), we can first obtain the CDF of $X(t)$ at time $t$ by the law of total probability as

$$
\begin{aligned}
& F_{X}(x, t) \\
& =P(X(t)<x) \\
& =\sum_{k=0}^{\infty} P\left(X_{a}(t)+X_{S}(t)<x \mid Z(t)=k\right) P(Z(t)=k),
\end{aligned}
$$

where $P(Z(t)=k)$ denotes the probability that $Z(t)=k$ occurs.

Denote the CDF of $X_{a}(t)$ as $U(x, t)$ and the PDF of $\bar{L}_{i}(i \in$ $\left.N^{+}\right)$as $f_{\bar{L}_{i}}^{<i>}\left(\bar{l}_{i}\right)$, where $\bar{L}_{i}$ is defined as $\sum_{k=0}^{Z(t)} L_{k} \mid Z(t)=i$ with realization $\bar{l}_{i}$. Then, (4) can be further transformed to be

$$
\begin{aligned}
F_{X}(x, t) & \\
= & U(x, t) \exp (-\tau t) \\
& \quad+\sum_{k=1}^{\infty} \int_{0}^{x} U\left(x-\bar{l}_{i}, t\right) f_{\bar{L}_{i}}^{<i>}\left(\bar{l}_{i}\right) d \bar{l}_{i} \frac{\exp (-\tau t)(\tau t)^{i}}{i !} .
\end{aligned}
$$

Equation (5) is a general form for $F_{X}(x, t)$. To get its analytical form, we choose the distribution of the shock damage size as $L_{k} \stackrel{\text { IID }}{\sim} N\left(\mu_{L}, \sigma_{L}^{2}\right)$. Then, $\bar{L}_{i} \sim N\left(i \mu_{L}, i \sigma_{L}^{2}\right)$. Taking the randomness of the degradation rate $\beta$ into account, the analytical form of $F_{X}(x, t)$ can be given by [18]

$$
\begin{aligned}
& F_{X}(x, t) \\
& =\Phi\left(\frac{x-\mu_{\beta} t}{\sigma_{\beta} t}\right) \exp (-\tau t) \\
& \quad+\sum_{i=1}^{\infty} \Phi\left(\frac{x-\left(\mu_{\beta} t+i \mu_{L}\right)}{\sqrt{\sigma_{\beta}^{2} t^{2}+i \sigma_{L}^{2}}}\right) \frac{\exp (-\tau t)(\tau t)^{i}}{i !}
\end{aligned}
$$

where $\Phi(\cdot)$ denotes the CDF of the standard normal distribution.

Accordingly, from (6), we can obtain the probability that no failure occurs before time $t$ as

$$
P(X(t)<w)=F_{X=w}(w, t) .
$$

Then, from (6) and (7), we can obtain the CDF of the lifetime $T$ as

$$
\begin{aligned}
F_{T}(t)= & 1-\Phi\left(\frac{w-\mu_{\beta} t}{\sigma_{\beta} t}\right) \exp (-\tau t) \\
& -\sum_{i=1}^{\infty} \Phi\left(\frac{w-\left(\mu_{\beta} t+i \mu_{L}\right)}{\sqrt{\sigma_{\beta}^{2} t^{2}+i \sigma_{L}^{2}}}\right) \frac{\exp (-\tau t)(\tau t)^{i}}{i !} .
\end{aligned}
$$

From (8), we can obtain the PDF of lifetime $T$ as

$$
\begin{aligned}
& f_{T}(t) \\
& =\frac{\partial}{\partial t} F_{T}(t)=\phi\left(\frac{w-\mu_{\beta} t}{\sigma_{\beta} t}\right) \frac{w}{\sigma_{\beta} t^{2}} \exp (-\tau t) \\
& +\lambda \Phi\left(\frac{w-\mu_{\beta} t}{\sigma_{\beta} t}\right) \exp (-\tau t) \\
& -\sum_{i=1}^{\infty}\left\{\phi\left(\frac{w-\left(\mu_{\beta} t+i \mu_{L}\right)}{\sqrt{\sigma_{\beta}^{2} t^{2}+i \sigma_{L}^{2}}}\right)\right. \\
& \frac{-\mu_{\beta} i \sigma_{L}^{2}+\left(-w+i \mu_{L}\right) \sigma_{\beta}^{2} t}{\left(\sigma_{\beta}^{2} t^{2}+i \sigma_{L}^{2}\right)^{3 / 2}} \\
& \frac{\exp (-\tau t)(\tau t)^{i}}{i !}+\Phi\left(\frac{w-\left(\mu_{\beta} t+i \mu_{L}\right)}{\sqrt{\sigma_{\beta}^{2} t^{2}+i \sigma_{L}^{2}}}\right) \\
& \left.\frac{\exp (-\tau t)(\tau t)^{i-1}\left(-\tau^{2} t+\tau i\right)}{i !}\right\} \text {, }
\end{aligned}
$$

where $\phi(\cdot)$ denotes the PDF of the standard normal distribution.

Remark 2. From (8) and (9), we can find that the CDF and PDF of the lifetime $T$ can be formulated analytically. Compared with the numerical results, such as the Monte Carlo simulation method [23], (8) and (9) can be implemented more quickly with less storage space. It can also be observed from (8) and (9) that there still exist some unknown parameters in the lifetime distributions. As mentioned above, for simplicity, these parameters were assumed to be known as prior information in literature, for example, [18]. However, if these parameters are misspecified in practice, then the obtained lifetime predictions may be unreasonable.

In the forthcoming section, we will present a dedicated parameter estimation procedure to estimate all these unknown parameters.

\section{Parameter Estimation}

The unknown parameters associated with the PDF and CDF of lifetime $T$ can be divided into the following three parts: (1) parameters in the abrupt damage part $X_{S}(t)$, including $\tau$ and $\xi=\left(\mu_{L}, \sigma_{L}\right)$; (2) parameters in the continuous degradation part $X_{a}(t)$, including $\mu_{\beta}$ and $\sigma_{\beta}$. For clarity, we denote all of these unknown parameters as $\Xi=\left(\tau, \mu_{L}, \sigma_{L}, \mu_{\beta}, \sigma_{\beta}\right)^{\prime}$ and the corresponding estimators as $\widehat{\Xi}=\left(\widehat{\tau}, \widehat{\mu}_{L}, \widehat{\sigma}_{L}, \widehat{\mu}_{\beta}, \widehat{\sigma}_{\beta}\right)^{\prime}$.

Assume that we have the degradation recordings of $M$ deteriorating systems of the same kind. Specifically, to obtain $\widehat{\Xi}$, the following three kinds of data are needed. First, the 
data of the system's total operating time and the total number of random shocks, denoted as $\left\{\left(W_{m}, s_{m}\right), m=1,2, \ldots, M\right\}$, where $W_{m}$ represents the total operating time of the $m$ th system, and $s_{m}$ represents the total number of random shocks of the $m$ th system during its operating time $W_{m}$. Second, shock damage size data, denoted as $\left\{l_{m, n}, m=1,2, \ldots, M ; n=\right.$ $\left.1,2, \ldots, s_{m}\right\}$, where $l_{m, n}$ is the damage size of the $n$th shock of the $m$ th system at time $t_{m, n}$. Third, degradation data, denoted as $\left\{x_{m, q}, m=1,2, \ldots, M ; q=1,2, \ldots, q_{m}\right\}$, where $x_{m, q}$ represents the $q$ th degradation data of the $m$ th deteriorating system at time $t_{m, q}$, and $q_{m}$ is the number of the observed degradation data of the $m$ th deteriorating system. Below, we will use these data to estimate the unknown parameters $\Xi$ via the MLE method.

4.1. Estimating of $\tau$. To estimate $\tau$, the following Lemma should be provided first.

Lemma 3 (see [23]). If $Z(t)$ is a Poisson process with rate $\tau$, as defined in (2), then the times between two random shocks follows an IID exponential distribution with mean $1 / \tau$.

Based on Lemma 3, we can formulate the log-likelihood function of $\left\{W_{m}, s_{m}\right\}$ in terms of $\tau$ as

$$
\Upsilon_{1}(\tau)=\sum_{m=1}^{M} \log \left(\tau^{s_{m}} e^{-\tau W_{m}}\right)
$$

Taking the derivative of $\Upsilon_{1}(\tau)$ with respect to $\tau$, and letting the result be equal to zero, we can finally obtain

$$
\widehat{\tau}=\frac{\sum_{m=1}^{M} s_{m}}{\sum_{m=1}^{M} W_{m}} .
$$

4.2. Estimating of $\mu_{L}$ and $\sigma_{L}$. We can estimate $\mu_{L}$ and $\sigma_{L}$ by the MLE method from $\left\{l_{m, n}\right\}$ via the following log-likelihood function

$$
\Upsilon_{2}\left(\mu_{L}, \sigma_{L}\right)=\sum_{m=1}^{M} \sum_{n=1}^{s_{m}} \log f\left(l_{m, n} ; \mu_{L}, \sigma_{L}\right)
$$

The estimators $\widehat{\mu}_{L}$ and $\widehat{\sigma}_{L}$ can be obtained by maximizing (11) in terms of $\mu_{L}$ and $\sigma_{L}$.

4.3. Estimating of $\mu_{\beta}$ and $\sigma_{\beta}$. Due to the existence of the abrupt damage part $X_{S}(t), \mu_{\beta}$ and $\sigma_{\beta}$ cannot be estimated from $\left\{x_{m, q}\right\}$ directly. In order to estimate these two unknown parameters, we have to transform the observed degradation data set $\left\{x_{m, q}\right\}$ first.

From (2), we have

$$
X(t)-\sum_{k=0}^{Z(t)} L_{k}=\alpha+\beta t
$$

where $\alpha=0$ as defined in (2).
Denoting the left hand side of (13) as $\Psi(t)$ (with its realization $\psi(t))$ yields

$$
\begin{aligned}
& \Psi(t)=X(t)-\sum_{k=0}^{Z(t)} L_{k}, \\
& \Psi(t)=\beta t .
\end{aligned}
$$

Based on (14), we can transform the observed degradation data set $\left\{x_{m, q}\right\}$ to be $\left\{\psi_{m, q}\right\}$ with the help of the shock damage size data $\left\{l_{m, n}\right\}$. The unknown parameters $\mu_{\beta}$ and $\sigma_{\beta}$ can be estimated from $\left\{\psi_{m, q}\right\}$ based on (15). For simplicity, we assume that $\left\{\psi_{m, q}\right\}$ from different deteriorating systems are independent, but $\left\{\psi_{m, q}\right\}$ of one deteriorating system at different times are dependent. Denote all of the transformed data of $\left\{\psi_{m, q}\right\}$ of the $m$ th system as $\left\{\psi_{m, 1: q_{m}}\right\}$. We have the following theorem.

Theorem 4 (see [24]). The transformed data $\left\{\psi_{m, 1: q_{m}}\right\}$ of the $m$ th system associated with (15) follows a multivariable normal distribution with mean and covariance:

$$
\begin{aligned}
& \boldsymbol{\mu}_{m}=\left(\mu_{\beta} t_{m, 1}, \mu_{\beta} t_{m, 2}, \ldots, \mu_{\beta} t_{m, q_{m}}\right)^{\prime}, \\
& \boldsymbol{\Sigma}_{m}=\sigma_{\beta}^{2} \mathbf{t}_{m, 1: q_{m}} \mathbf{t}_{m, 1: q_{m}}^{\prime},
\end{aligned}
$$

respectively, where $\mathbf{t}_{m, 1: q_{m}}=\left(t_{m, 1}, t_{m, 2}, \ldots, t_{m, q_{m}}\right)^{\prime}$ representing the times associated with $\left\{\psi_{m, 1: q_{m}}\right\}$.

Based on Theorem 4, the log-likelihood function of all the transformed degradation data, $\left\{\psi_{m, q}, m=1,2, \ldots, M ; q=\right.$ $\left.1,2, \ldots, q_{m}\right\}$, can be formulated as

$$
\begin{aligned}
\Upsilon_{3}\left(\mu_{\beta}, \sigma_{\beta}\right) \\
=-\frac{\sum_{m=1}^{M} q_{m}}{2} \log (2 \pi)-\frac{1}{2} \sum_{m=1}^{M} \log \left|\Sigma_{m}\right| \\
\quad-\frac{1}{2} \sum_{m=1}^{M}\left(\psi_{m, 1: q_{m}}-\boldsymbol{\mu}_{m}\right)^{\prime} \Sigma_{m}{ }^{-1}\left(\psi_{m, 1: q_{m}}-\boldsymbol{\mu}_{m}\right) .
\end{aligned}
$$

Maximize (18), the estimators of $\mu_{\beta}$ and $\sigma_{\beta}$ can be obtained.

Till now, all of the unknown parameters $\Xi$ are estimated. Substituting the estimated $\widehat{\Xi}$ into (8) and (9), we can obtain the CDF and PDF of the lifetime $T$ for this class of deteriorating systems subjected to both continuous smooth degradation and abrupt damage. Next, we will apply the obtained lifetime prediction results to a sample of simulated data.

\section{Illustrative Example}

This section is devoted to providing an illustrative example to demonstrate the feasibility and superiority of our proposed lifetime prediction method. For comparison, we choose the method in [18] as the rival model, which is also termed as Peng's approach hereafter. We first generate some data as the basis for demonstration. 
TABLE 1: The settings of the parameters.

\begin{tabular}{ccccccc}
\hline$\alpha$ & $\mu_{\beta}$ & $\sigma_{\beta}$ & $\mu_{L}$ & $\sigma_{L}$ & $\tau$ & $\Delta t$ \\
\hline 0 & 0.4 & 0.1 & 15 & 1 & 0.05 & 1 \\
\hline
\end{tabular}

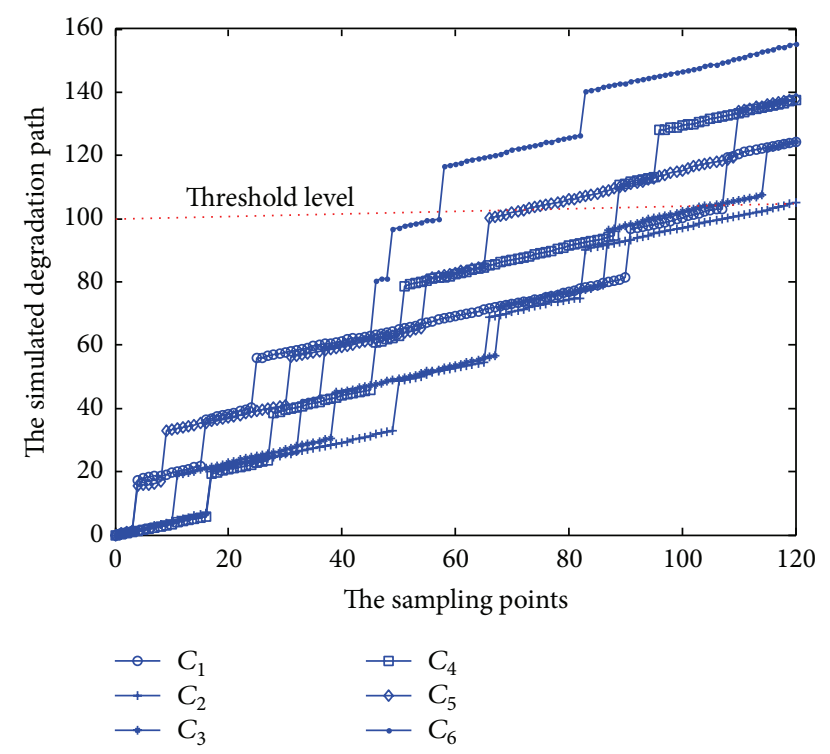

FIGURE 1: The simulated degradation paths.

5.1. Data Generation. The data are simulated by (2) with the parameters as shown in Table 1 , where $\Delta t$ is the discretization step. Based on these settings, six degradation paths are simulated with 120 samples of points (except $X(0)=0$ ) in each degradation path, as illustrated in Figure 1. For clarity, we denote these simulated degradation paths from the first to the sixth path with $C_{\gamma}(\gamma=1, \ldots, 6)$, respectively. From Figure 1, it can be observed that the degradation path evolves increasingly due to both the continuous smooth degradation and additional abrupt damage. We also provide the information of the shocks associated with these six degradation paths in Table 2, where the first quantity in each parenthesis denotes the occurring time of each shock, and the second quantity denotes the damage size of the shock on system's degradation.

5.2. Parameters Estimation and Assumptions Testing. In this subsection, we focus on estimating the unknown parameters associated with the degradation model, based on the simulated degradation paths above, that is, $C_{1} \sim C_{6}$. The estimated parameters are shown in Table 3. From Table 3, we can find that the estimated parameters are all very close to their real values as we used before. For a practical case, it is necessary to test the reasonableness of the assumptions used in the shock related part of the degradation model based on the statistical hypothesis testing methods [25]. Specifically, the following two assumptions are tested mathematically. First, the Poisson process assumption for the arrival of the random shocks by the Kolmogorov-Smirnov test. Second, the normality assumption of the damage sizes of random shocks on system's degradation via the Lilliefors test. The testing results accept both of these two assumptions at the

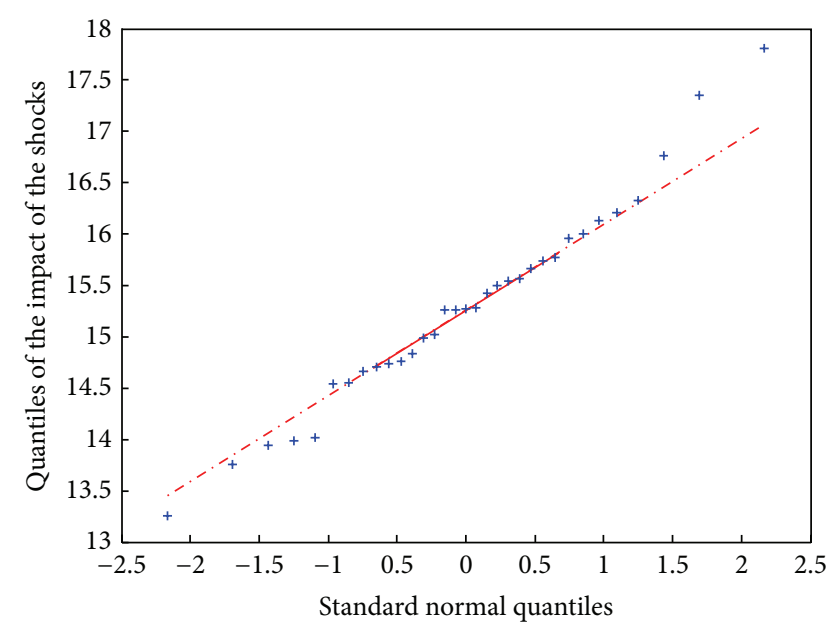

FIGURE 2: QQ plot of the damage sizes of the shocks versus standard normal distribution.

$5 \%$ significance level. In addition, to provide an intuitive illustration, the Quantile-Quantile (QQ) plot of the normality assumption of the damage sizes of random shocks versus the standard normal distribution is further plotted in Figure 2. Observed from Figure 2, the QQ plot is close to being linear, which reveals the rationality of the normal distribution assumptions for the damage sizes of the shocks on system degradation.

5.3. Predictions of the PDF of the Lifetime Based on the Estimated Parameters. This subsection will apply the proposed lifetime prediction in this paper to the simulated data to validate its effectiveness. Specifically, the unknown parameters in the lifetime distributions in (8) and (9) are replaced by the estimators in Table 2 , and the threshold level is set to be $w=100$. For comparison, Peng's approach in [18] is also applied to the simulated data. As mentioned above, the unknown parameters in [18] are specified by the prior information or subjective experiences, and thus, it is likely that the parameters are misspecified. However, once these parameters are misspecified in Peng's approach, the predicted lifetime may be unreasonable. Below, we will investigate the influence of the misspecification of the mean of $\beta$ (i.e., $\mu_{\beta}$ ), the mean of shock damage size $L_{k}$ (i.e., $\mu_{L}$ ), and the arrival rate of the random shocks, $\tau$, on the lifetime predictions in succession. Here the term "misspecification" means that the unknown parameters are appointed much smaller or larger compared to the real values due to the imprecision of the prior information or the subjective experiences. By employing Monte Carlo simulation method [23], we also plot the empirical lifetime distributions (can be viewed as the real lifetime distributions) based on the real parameter values in Table 1, to facilitate the comparison process. Specifically, the simulated run of the Monte Carlo method is set to be 100000 .

5.3.1. The Parameter $\mu_{\beta}$ Is Misspecified. We first explore the lifetime prediction results when $\mu_{\beta}$ is misspecified in Peng's approach. The empirical lifetime distributions, the predicted 
TABLE 2: The occurring times and damage sizes of the shocks in the simulated paths.

\begin{tabular}{|c|c|c|c|c|c|c|c|}
\hline & 1st shock & 2nd shock & 3rd shock & 4 th shock & 5th shock & 6th shock & 7th shock \\
\hline$C_{1}$ & $(3,16.3275)$ & $(15,14.5469)$ & $(24,15.6652)$ & $(90,15.2858)$ & $(107,16.2108)$ & & \\
\hline $\mathrm{C}_{2}$ & $(16,13.2536)$ & $(49,15.5015)$ & $(65,14.5447)$ & $(82,15.2710)$ & & & \\
\hline$C_{3}$ & $(10,14.7404)$ & $(38,14.7076)$ & $(67,15.4248)$ & $(86,17.3549)$ & $(114,14.7640)$ & & \\
\hline$C_{4}$ & $(16,13.7604)$ & $(27,14.8355)$ & $(45,14.9892)$ & $(50,15.7752)$ & $(88,16.0038)$ & $(95,15.0241)$ & \\
\hline$C_{5}$ & $(3,14.0170)$ & $(8,16.1365)$ & $(30,15.2565)$ & $(54,15.5600)$ & $(65,15.2636)$ & $(109,14.6566)$ & \\
\hline $\mathrm{C}_{6}$ & $(16,13.9385)$ & $(32,15.9557)$ & $(36,15.5479)$ & $(45,17.8065)$ & $(48,15.7366)$ & $(57,16.7623)$ & $(82,13.9892)$ \\
\hline
\end{tabular}
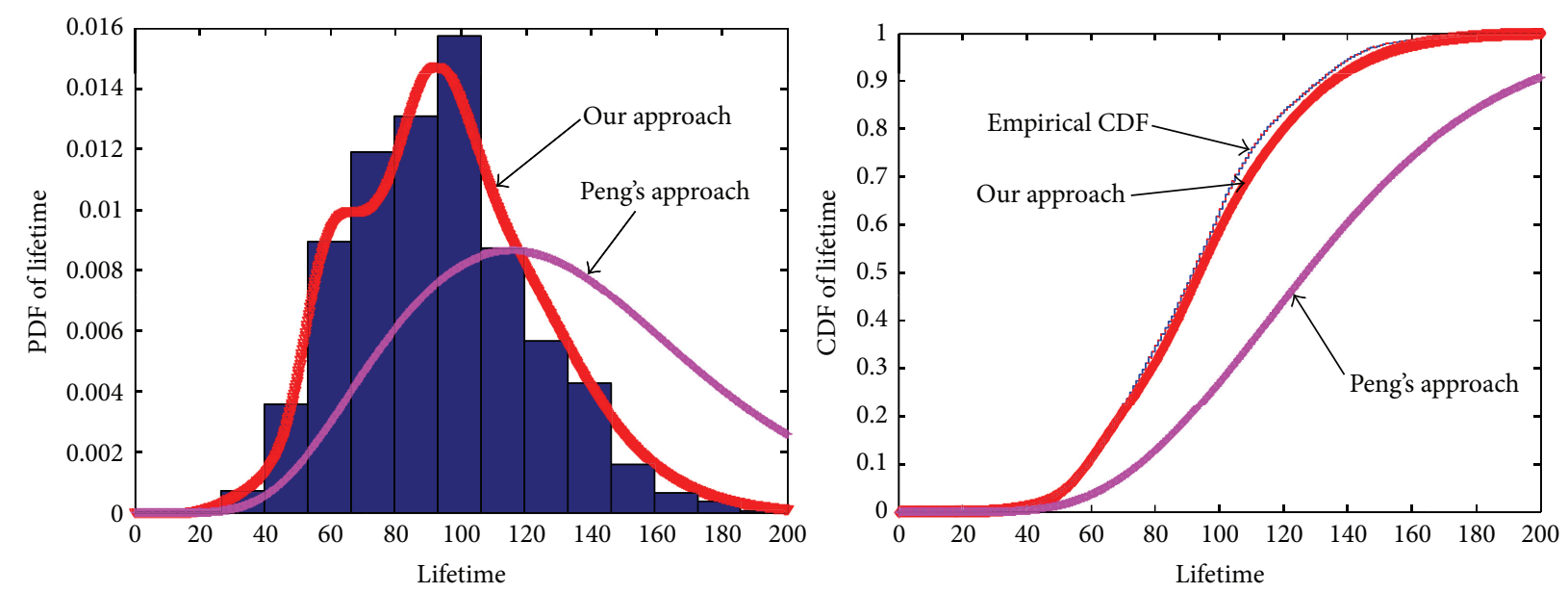

(a) Peng's approach with a smaller $\mu_{\beta}=0.1$
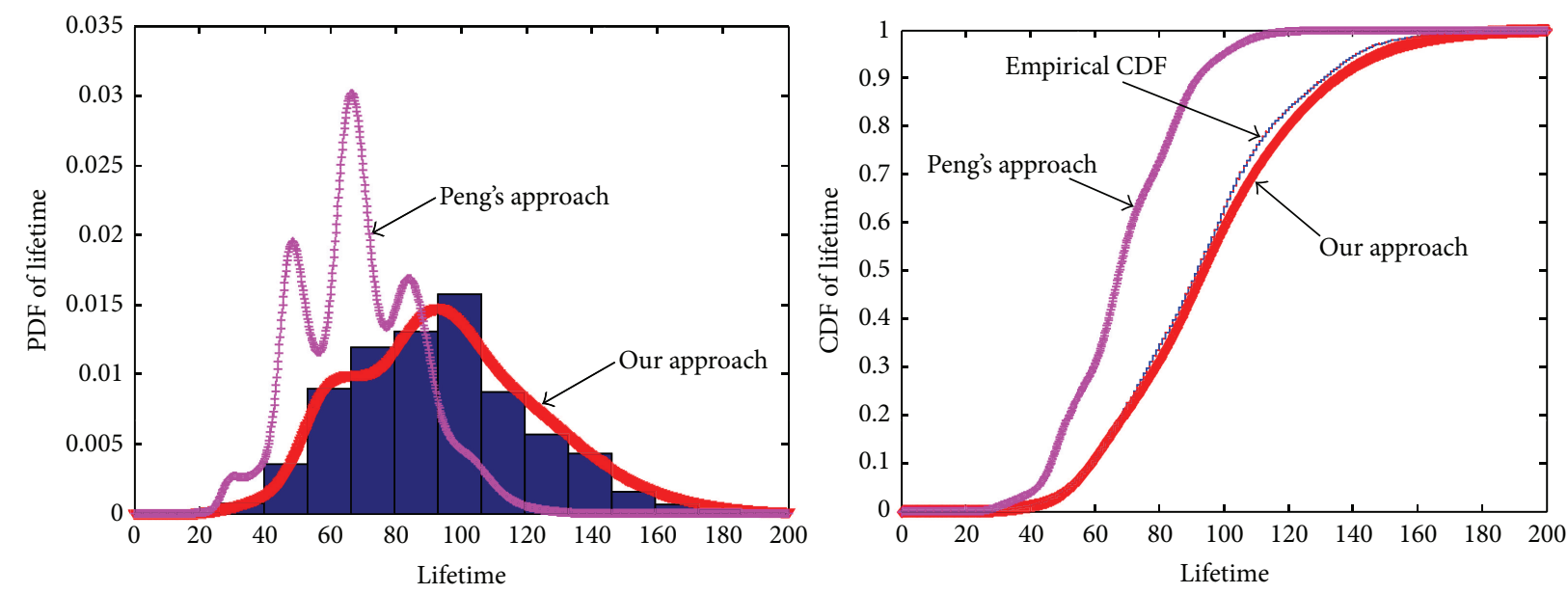

(b) Peng's approach with a larger $\mu_{\beta}=0.8$

FIgURE 3: Predicted PDF and CDF of the lifetime when $\mu_{\beta}$ is misspecified in Peng's approach.

TABLE 3: The estimated parameters.

\begin{tabular}{lcccc}
\hline$\widehat{\mu}_{\beta}$ & $\widehat{\sigma}_{\beta}$ & $\widehat{\mu}_{L}$ & $\widehat{\sigma}_{L}$ & $\widehat{\tau}$ \\
\hline 0.3897 & 0.0512 & 15.4547 & 0.6439 & 0.0458 \\
\hline
\end{tabular}

lifetime distributions of our approach, and the predicted lifetime distributions of Peng's approach with specified $\mu_{\beta}$ are plotted in Figure 3. Specifically, Figures 3(a) and 3(b) show the predicted results associated with a much smaller $\mu_{\beta}$ with $\mu_{\beta}=0.1$ and a much larger $\mu_{\beta}$ with $\mu_{\beta}=0.8$ than the real value $\mu_{\beta}=0.4$ in Peng's approach, respectively.

From Figure 3, the following two observations can be obtained. First, the PDF and CDF of the lifetime predicted by our approach can fit the empirical lifetime distributions better than those predicted by Peng's approach with misspecified $\mu_{\beta}$. Second, the predicted lifetime of PDF by Peng's approach in Figure 3(a) is much larger than the empirical PDF (i.e., the histogram), and the CDF of Peng's approach increases much slower than the empirical CDF. The opposition can be 

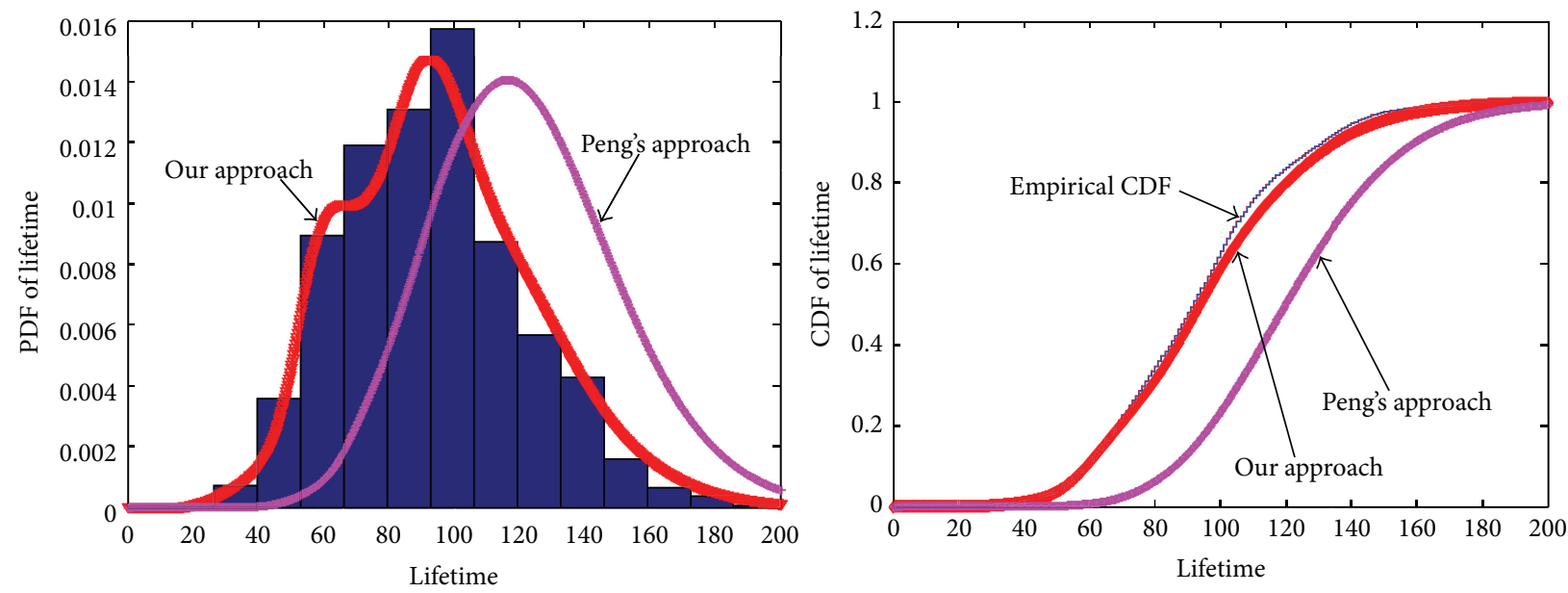

(a) Peng's approach with a smaller $\mu_{L}=10$
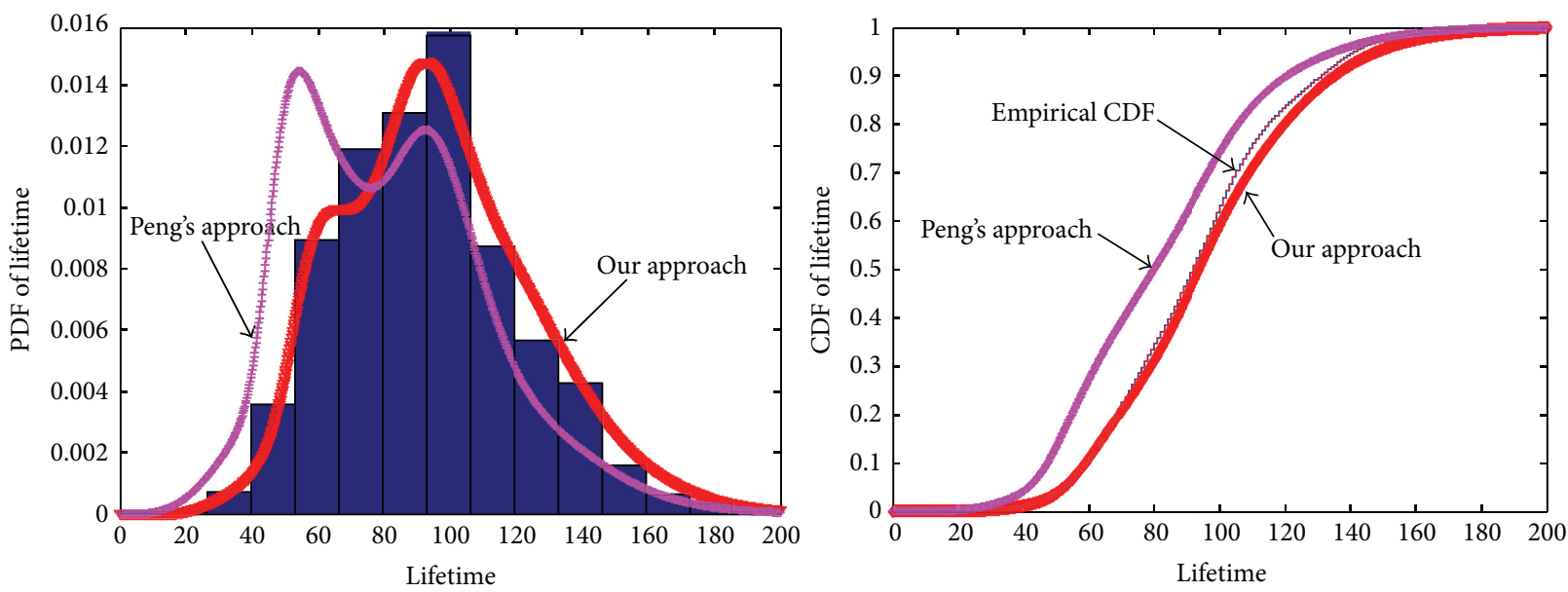

(b) Peng's approach with a larger $\mu_{L}=20$

Figure 4: Predicted PDF and CDF of the lifetime when $\mu_{L}$ is misspecified in Peng's approach.

observed when $\mu_{\beta}$ is chosen larger than the real value $\mu_{\beta}=0.4$ in Figure 3(b). This is intuitive since a smaller (larger) $\mu_{\beta}$ corresponds to a lower (higher) mean degradation rate of the deteriorating systems.

5.3.2. The Parameter $\mu_{L}$ Is Misspecified. We further investigate the influence of the mean of the shock damage size, $\mu_{L}$, on the predicted lifetime distributions. The $\mu_{L}$ is chosen to be 10 and 20, which are, respectively, much smaller and larger than the real value $\mu_{L}=15$, as shown in Table 1 . The comparison results are plotted in Figure 4.

As indicated in Figure 4(a), both PDF and CDF of the lifetime predicted by Peng's approach with a smaller $\mu_{L}=10$ are translated a lot to the right hand side of the empirical distributions. This implies that the lifetime will be overestimated when the mean of the shock damage size, $\mu_{L}$, is chosen to be a much smaller value than its real value. In contrary, the opposite can be observed when $\mu_{L}$ is misspecified to be a much larger value than the real one, as shown in Figure 4(b). Differently, the lifetime distributions predicted by our approach can track the empirical ones well, which reveals a more accurate lifetime predicting result than Peng's approach with misspecified $\mu_{L}$.

5.3.3. The Parameter $\tau$ Is Misspecified. We now proceed to study the lifetime prediction results when the arrival rate of the random shocks is misspecified in Peng's approach. Specifically, the arrival rate of the random shocks is set to be $\tau=0.02$ and $\tau=0.06$, which correspond to a much smaller and larger arrival rate than the real value $\tau=0.04$ in Table 1 . The predicted PDF of CDF of the lifetime associated with these two cases is shown in Figure 5.

From Figure 5, the following three aspects can be observed. First, the predicted PDF and CDF predicted by our approach can track the empirical ones well. Second, when the arrival rate $\tau$ is misspecified to a much smaller value, both PDF and CDF of the lifetime predicted by Peng's approach will be right translated, as shown in Figure 5(a). Third, when the arrival rate $\tau$ is misspecified to a much larger value, both PDF and CDF of the lifetime predicted by Peng's approach will be left translated, as shown in Figure 5(b). This phenomenon is natural since a smaller (larger) arrival 

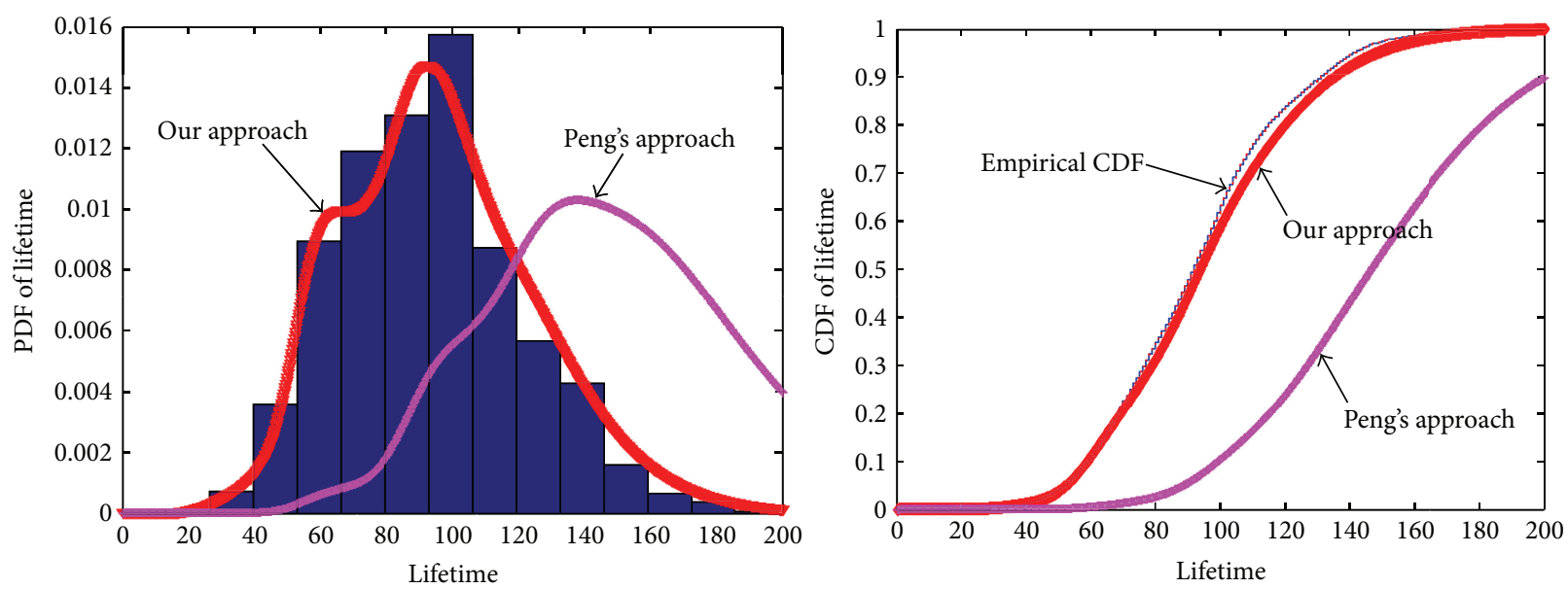

(a) Peng's approach with a smaller $\tau=0.02$
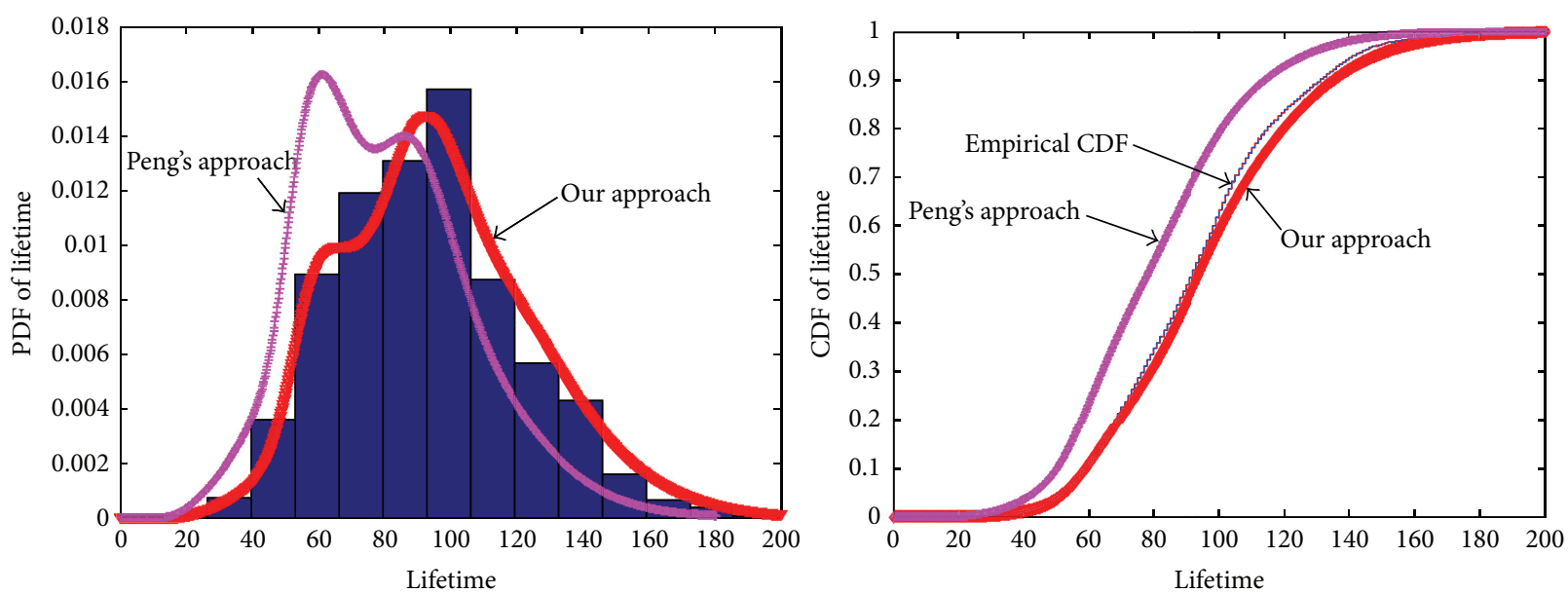

(b) Peng's approach with a larger $\tau=0.06$

FIgure 5: Predicted PDF and CDF of the lifetime when $\tau$ is misspecified in Peng's approach.

rate of the random shocks corresponds to a higher (lower) arrival frequency of the random shocks, thereby expediting or slowing the arrival time of failures.

In summary, we can conclude this experiment with the following two results. First, the predicted lifetime distributions by our approach fit well with the empirical lifetime distributions. Second, the predicted lifetime distributions deviate far from the empirical lifetime distributions by Peng's approach with misspecified parameter settings.

\section{Conclusions and Future Researches}

This paper was concerned with the lifetime prediction issue of a class of deteriorating systems subjected to both continuous smooth degradation and abrupt damage due to random shocks. To obtain more accurate lifetime predictions, a dedicated parameter estimation procedure was developed for the unknown parameters in the degradation model. An illustrative example was provided to demonstrate the effectiveness and superiority of the newly proposed lifetime prediction method, which revealed that the predicted lifetime distributions by our approach can increase the lifetime prediction accuracy compared to those obtained by the rival approach in literature.

This paper is just a pilot study for the deteriorating systems subjected to both continuous smooth degradation and abrupt damage. There still exist some uncovered scopes that can be further investigated in future. First, the continuous smooth degradation part is assumed to be modeled by a linear degradation model; however, nonlinearity exists extensively in practice. Predicting the lifetime distributions of the deteriorating systems subjected to both nonlinearitydriven continuous degradation and the abrupt damage of random shocks is more general. Second, this paper focuses on the lifetime prediction issue of a kind of deteriorating systems. For a specific deteriorating system in service, it is much more desirable to develop a real-time parameter estimation and lifetime update method [26-28]. Third, we only study the lifetime prediction issue in this paper. The operations management problems (such as inventory controlling and maintenance scheduling) $[8,9]$ based on the predicted lifetime distributions in this paper are also future directions for us. 


\section{Conflict of Interests}

The authors declare that there is no conflict of interests regarding the publication of this paper.

\section{Acknowledgments}

The research reported here is partially supported by NSFC under Grant no. 51475462 and the advanced research project under Grant no. 9140A19030314JB47276.

\section{References}

[1] M. Pecht, Prognostics and Health Management of Electronics, John Wiley \& Sons, Hoboken, NJ, USA, 2008.

[2] M. Dong, "A tutorial on nonlinear time-series data mining in engineering asset health and reliability prediction: concepts, models, and algorithms," Mathematical Problems in Engineering, vol. 2010, Article ID 175936, 22 pages, 2010.

[3] G. Li, S. J. Qin, Y. D. Ji, and D. H. Zhou, "Reconstruction based fault prognosis for continuous processes," Control Engineering Practice, vol. 18, no. 10, pp. 1211-1219, 2010.

[4] E. Zio, "Reliability engineering: old problems and new challenges," Reliability Engineering \& System Safety, vol. 94, no. 2, pp. 125-141, 2009.

[5] E. Zio and A. Zoia, "Parameter identification in degradation modeling by reversible-jump Markov Chain Monte Carlo," IEEE Transactions on Reliability, vol. 58, no. 1, pp. 123-131, 2009.

[6] E. Zio and M. Compare, "Evaluating maintenance policies by quantitative modeling and analysis," Reliability Engineering and System Safety, vol. 109, pp. 53-65, 2013.

[7] X.-S. Si, W. Wang, C.-H. Hu, M.-Y. Chen, and D.-H. Zhou, “A Wiener-process-based degradation model with a recursive filter algorithm for remaining useful life estimation," Mechanical Systems and Signal Processing, vol. 35, no. 1-2, pp. 219-237, 2013.

[8] Z.-Q. Wang, W. Wang, C.-H. Hu, X.-S. Si, and W. Zhang, "A prognostic-information-based order-replacement policy for a non-repairable critical system in service," IEEE Transactions on Reliability, 2014.

[9] Z. Q. Wang, C. H. Hu, W. Wang, X. Y. Kong, and W. Zhang, "A prognostics-based spare part ordering and system replacement policy for a deteriorating system subjected to a random lead time," International Journal of Production, 2014.

[10] Z. Q. Wang, C. H. Hu, W. Wang, Z. J. Zhou, and X. S. Si, "A case study of remaining storage life prediction using stochastic filtering with the influence of condition monitoring," Reliability Engineering \& System Safety, vol. 132, pp. 186-195, 2014.

[11] X.-S. Si, W. Wang, C.-H. Hu, and D.-H. Zhou, "Remaining useful life estimation-a review on the statistical data driven approaches," European Journal of Operational Research, vol. 213, no. 1, pp. 1-14, 2011.

[12] X.-S. Si, W. Wang, M.-Y. Chen, C.-H. Hu, and D.-H. Zhou, "A degradation path-dependent approach for remaining useful life estimation with an exact and closed-form solution," European Journal of Operational Research, vol. 226, no. 1, pp. 53-66, 2013.

[13] Z.-Q. Wang, C.-H. Hu, W. Wang, and X.-S. Si, "An additive Wiener process-based prognostic model for hybrid deteriorating systems," IEEE Transactions on Reliability, vol. 63, no. 1, pp. 208-222, 2014.
[14] C. J. Lu and W. Q. Meeker, "Using degradation measures to estimate a time-to-failure distribution," Technometrics, vol. 35, no. 2, pp. 161-174, 1993.

[15] N. Z. Gebraeel, M. A. Lawley, R. Li, and J. K. Ryan, "Residual-life distributions from component degradation signals: a Bayesian approach," IIE Transactions (Institute of Industrial Engineers), vol. 37, no. 6, pp. 543-557, 2005.

[16] S. J. Bae and P. H. Kvam, "A nonlinear random-coefficients model for degradation testing," Technometrics, vol. 46, no. 4, pp. 460-469, 2004.

[17] Z. Wang, W. Wang, C. Hu, X. Si, and J. Li, "A real-time prognostic method for the drift errors in the inertial navigation system by a nonlinear random-coefficient regression model," Acta Astronautica, vol. 103, pp. 45-54, 2014.

[18] H. Peng, Q. Feng, and D. W. Coit, "Reliability and maintenance modeling for systems subject to multiple dependent competing failure processes," IIE Transactions, vol. 43, no. 1, pp. 12-22, 2010.

[19] L. Jiang, Q. Feng, and D. W. Coit, "Reliability and maintenance modeling for dependent competing failure processes with shifting failure thresholds," IEEE Transactions on Reliability, vol. 61, no. 4, pp. 932-948, 2012.

[20] E. Keedy and Q. Feng, "Reliability analysis and customized preventive maintenance policies for stents with stochastic dependent competing risk processes," IEEE Transactions on Reliability, vol. 62, no. 4, pp. 887-897, 2013.

[21] S. Song, D. W. Coit, Q. Feng, and H. Peng, "Reliability analysis for multi-component systems subject to multiple dependent competing failure processes," IEEE Transactions on Reliability, vol. 63, no. 1, pp. 331-345, 2014.

[22] K. Rafiee, Q. Feng, and D. W. Coit, "Reliability modeling for dependent competing failure processes with changing degradation rate," IIE Transactions, vol. 46, no. 5, pp. 483-496, 2014.

[23] S. M. Ross, Introduction to Probability Models, Academic Press, Oxford, UK, 9th edition, 2007.

[24] Z. Q. Wang, C. H. Hu, W. Wang, and G. J. Dong, "Wiener process-based online prediction method of remaining useful life for draught fans in steel mills," Journal of University of Science and Technology Beijing, vol. 36, no. 10, pp. 1361-1368, 2014.

[25] Y. Zhao, Data Analysis of Reliability, National Defense Industry Press, Beijing, China, 2011.

[26] Z.-J. Zhou, C.-H. Hu, D.-L. Xu, M.-Y. Chen, and D.-H. Zhou, "A model for real-time failure prognosis based on hidden Markov model and belief rule base," European Journal of Operational Research, vol. 207, no. 1, pp. 269-283, 2010.

[27] Z.-J. Zhou, C.-H. Hu, X.-X. Han, H.-F. He, X.-D. Ling, and B.C. Zhang, "A model for online failure prognosis subject to two failure modes based on belief rule base and semi-quantitative information," Knowledge-Based Systems, vol. 70, pp. 221-230, 2014.

[28] Z.-J. Zhou, C.-H. Hu, J.-B. Yang, D.-L. Xu, and D.-H. Zhou, "Online updating belief rule based system for pipeline leak detection under expert intervention," Expert Systems with Applications, vol. 36, no. 4, pp. 7700-7709, 2009. 


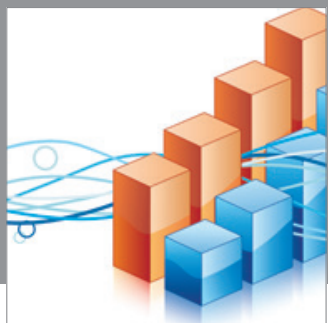

Advances in

Operations Research

mansans

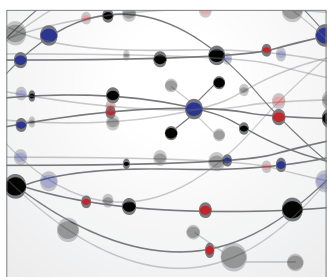

The Scientific World Journal
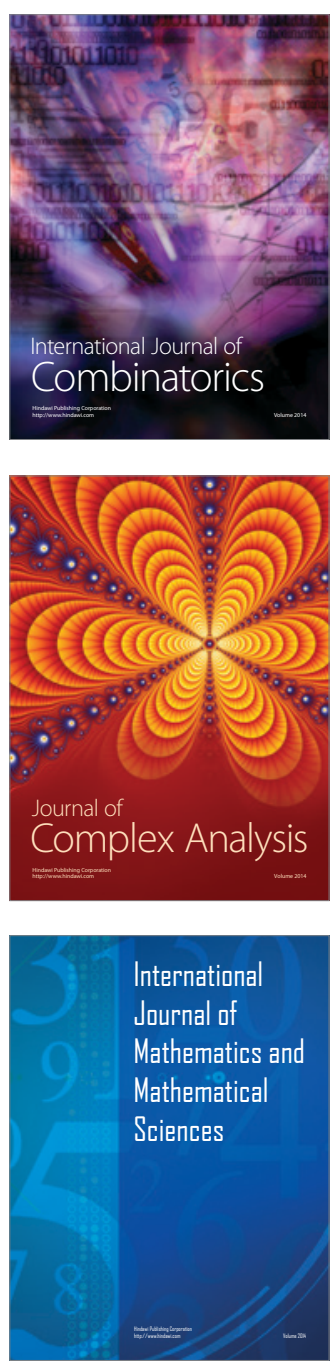
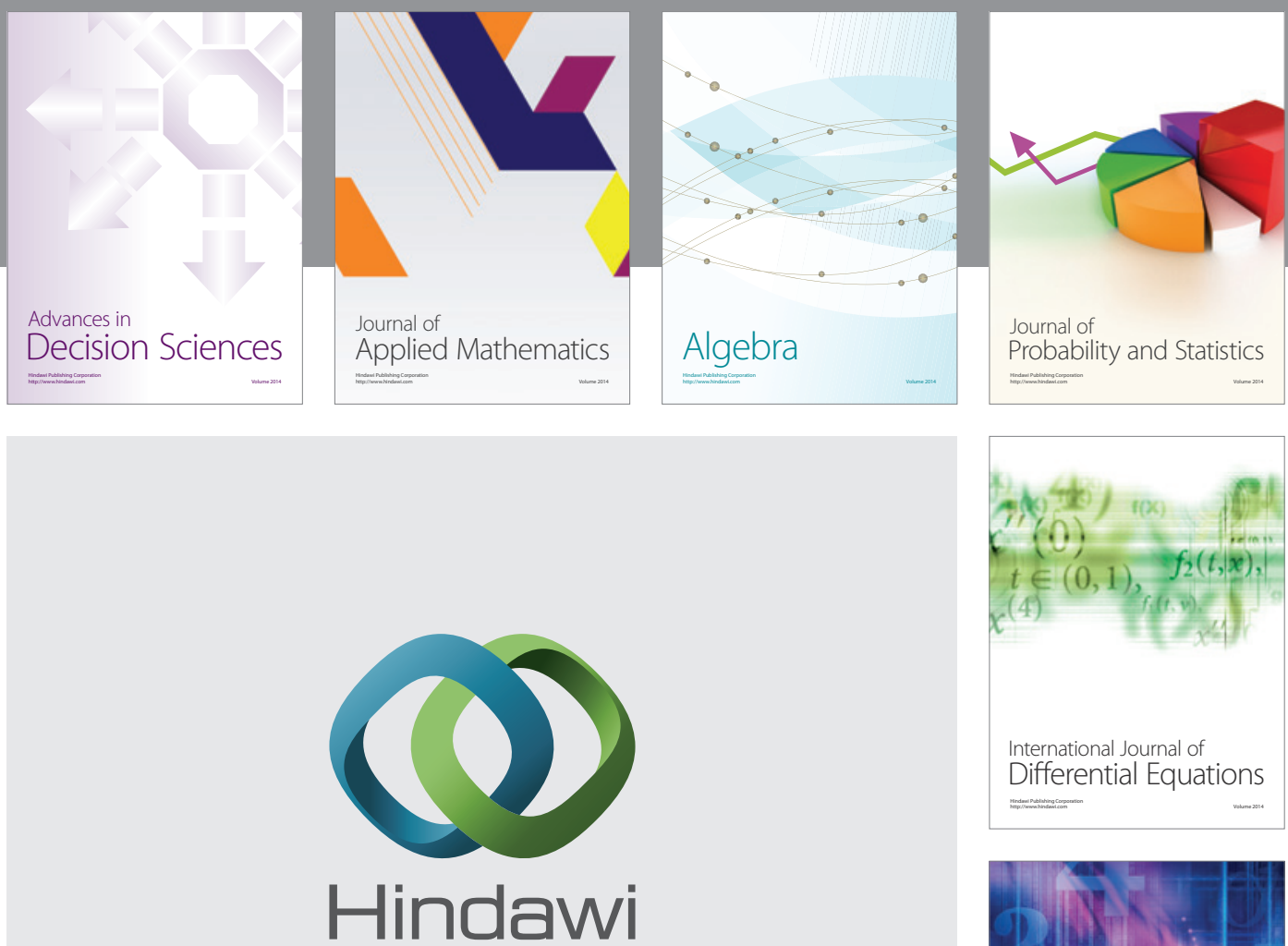

Submit your manuscripts at http://www.hindawi.com
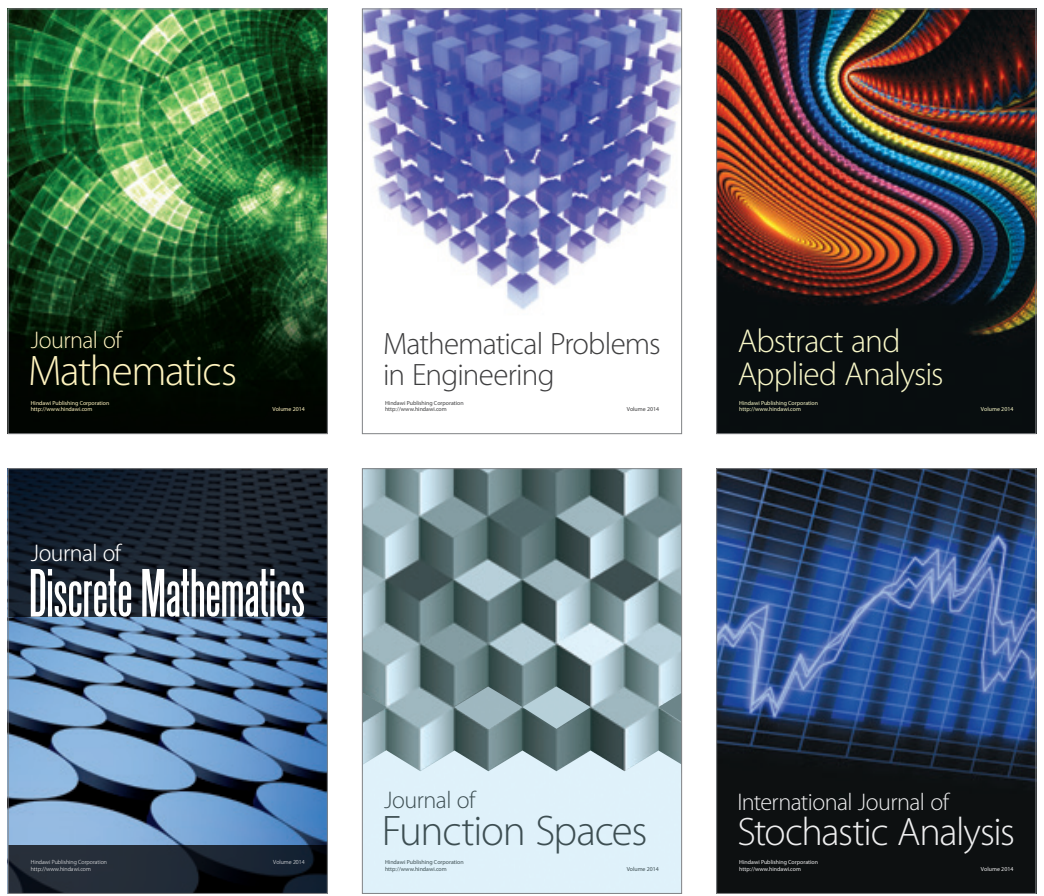

Journal of

Function Spaces

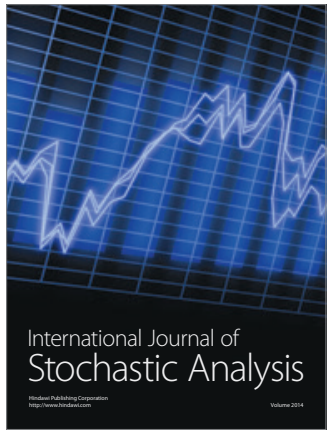

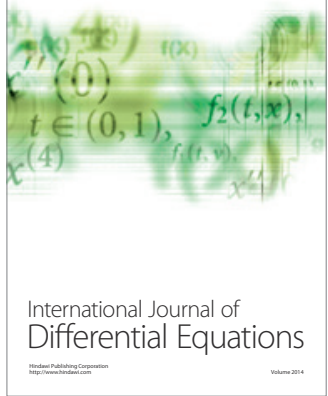
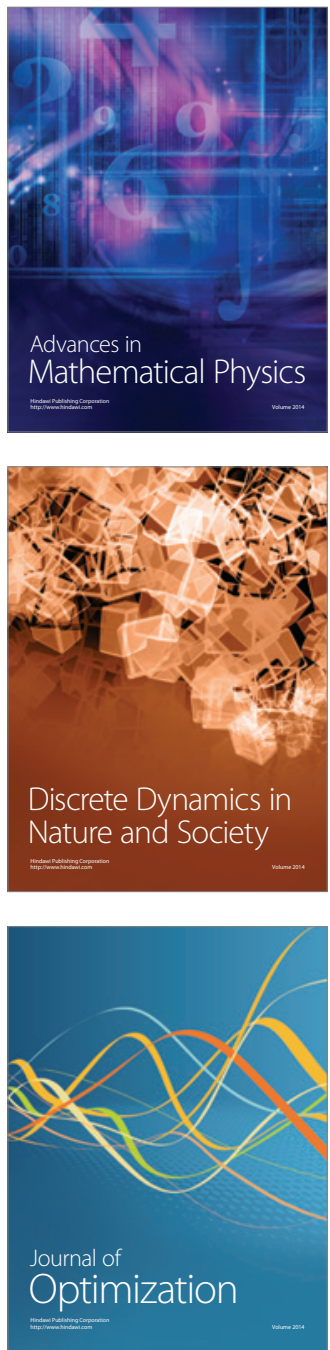